\title{
CONSIDERACIONES IGNACIANAS PARA UNA ÉTICA DE LA COMUNICACIÓN
}

\section{IGNATIAN CONSIDERATIONS FOR ETHICS OF COMMUNICATION}

\section{CARLOS I. MAN-GING VILLANUEVA ${ }^{1}$ MYRIAM MERCHÁN BARROS ${ }^{2}$}

Recibido: 31 de enero de 2017 Aceptado: 6 de marzo de 2017

\footnotetext{
${ }^{1}$ Pontificia Universidad Católica del Ecuador, Facultad Eclesiástica de Ciencias Filosófico-Teológicas, Escuela de Lengua y Literatura, Escuela de Teología, Quito, Ecuador (cimanging@puce.edu.ec).

${ }^{2}$ Pontificia Universidad Católica del Ecuador, Facultad de Comunicación, Lingüística y Literatura, Escuela de Lengua y Literatura (mmerchan@puce.edu.ec).
} 



\title{
CONSIDERACIONES IGNACIANAS PARA UNA ÉTICA DE LA COMUNICACIÓN
}

\section{IGNATIAN CONSIDERATIONS FOR ETHICS OF COMMUNICATION}

\author{
CARLOSI. MAN-GING VILLANUEVA, MYRIAM MERCHÁN BARROS
}

\begin{abstract}
PALABRAS CLAVE: ética de la responsabilidad, espiritualidad ignaciana, comunicación, trascendencia.
\end{abstract}

KEY WORDS: ethics of responsibility, Ignatian spirituality, communication, transcendence.

\section{RESUMEN}

Se aborda la evolución histórica de la propuesta ignaciana de educación y en particular del cuidado del lenguaje en el proceso formativo permanente para hacernos personas. El lenguaje, eje transversal que atraviesa las diversas disciplinas, integra el conocimiento con una visión holística y sistémica del mundo. La consideración de tiempos, lugares y personas proporciona al sistema educativo una dimensión de flexibilidad para crear juntos, tanto el docente como el estudiante, un clima de discernimiento y excelencia.

La propuesta ignaciana de educación realza la importancia del lenguaje para el desarrollo de la autorreflexión y la reflexión necesarias para entender el mundo, para discernir -implicación del pensamiento y el lenguaje- y pasar a la trascendencia en la experiencia, en la puesta en práctica y en el testimonio de nuestras acciones desde el lenguaje, para no olvidar la obligación ética de buscar y difundir la verdad. 


\begin{abstract}
We approach the historical evodiscernment and excellence. lution of the Ignatian proposal of education and in particular the care of the language in the permanent formative process to make us persons. Language, as a transversal axis that crosses various disciplines, integrates knowledge with a holistic and systemic view of the world. The considerations of times, places and people provides the educational system with a flexible dimension to create, tea-

The Ignatian proposal of education emphasizes the importance of language to reach self-reflection and reflection, needed to understand the world, to discern -where thought and language are present- and to pass to transcendence in experience, implementation and testimony of our actions by language, so as not to forget the ethical obligation to seek and spread the truth.
\end{abstract} cher and student together, a climate of

\title{
CONSIDERACIONES IGNACIANAS PARA UNA ÉTICA DE LA COMUNICACIÓN
}

"El objetivo de la educación jesuita es ayudar al desarrollo más completo posible de todos los talentos dados por Dios a cada individuo como miembro de la comunidad humana".

\section{INTRODUCCIÓN}

El fundador de la Pontificia Universidad Católica del Ecuador, P. Aurelio Espinosa Pólit, S.J., proponía a mediados del siglo XX como único examen de ingreso a la universidad el saber leer y escribir bien. Dicha intuición no es vana, sino más bien acertada, puesto que dichas competencias genéricas son las que garantizan el éxito de la formación humana y profesional de las personas, pues aúnan las competencias instrumentales, las interpersonales individuales, las interpersonales sociales y las sistémicas (Villa y Poblete, 2005; Villa \& García, 2014). ¿En dónde radica esta certeza y en qué procesos se fundamenta la mis-

${ }^{1}$ Características de la Educación de la Compañía de Jesús, n. 25. 
ma? ¿Qué elementos de la adquisición y manejo del lenguaje son los que la posibilitan, y en qué medida favorecen una ética de la comunicación? Estas preguntas son las que, en el marco del jubileo de los setenta años de la fundación de esta universidad confiada a la Compañía de Jesús en el Ecuador, dirigen nuestro trabajo. Esperamos que el desarrollo de estas consideraciones ignacianas sea de interés para el público lector.

Según la etimología de la palabra que encabeza el título de esta reflexión, el verbo latino considerare derivado de sidus, sideris (estrella), indica la acción de mirar junto con las estrellas. De esta manera se puede contemplar un fenómeno en el horizonte o plano del infinito, con el objetivo de examinarlo atentamente. Su utilidad se remonta a la antigüedad en las culturas caldea y babilónica, y hacía referencia a mirar los astros para predecir el destino. Su evolución ha llevado al sentido más preciso de pensar sobre algo u opinar, para reflexionar con atención y cuidado, dada la importancia del asunto en cuestión.

El autor de los Ejercicios Espirituales (Arzubialde, 2009) utiliza efectivamente el verbo "considerar" en los pasajes de mayor importancia para el provecho espiritual de los ejercitantes: ... mucho se ha de considerar... es la frase reiterada en las meditaciones de la elección de estado (EE 135), para enmendary reformar la propia vida y estado (189), la contemplación para alcanzar amor (240), o en el ministerio de distribuir limosnas (337). Son los textos de gran relevancia en que el ejercitante es invitado a discernir en libertad para conocerse mejor y servir al Señor Jesucristo, al Padre y al Espíritu Santo.

El presupuesto inicial de los Ejercicios Espirituales es "salvar la proposición del prójimo" (EE 23), como una disposición fundamental para comenzar este itinerario. Esta actitud es a la vez una condición esencial de la ética de la responsabilidad para la comunicación. Comunicar es vivir la dimensión ética del ser social. Su ejercicio implica una reflexión profunda a fin de crear una verdadera comunidad de la comunicación. Buscar su fundamento antropológico puede llegar incluso a la audacia de lo imposible, puesto que para algunos teóricos "la auténtica dificultad de la ética se encuentra en su fundamentación" (Lellouche, 2001, 200).

Lo que se propone en este estudio, en forma sucinta, es el aporte de algunas consideraciones ignacianas desde el horizonte de la espiritualidad y la praxis educativa universitaria. Más que aprender algo, interesa "aprender a aprender" fortaleciendo los principales procesos cognitivos (cfr. Villa, 2011; Poblete et alt., 2016). De aquí la insistencia del desarrollo de ciertas competencias genéricas, entre las que 
destacamos las instrumentales -cognitivas, tecnológicas y lingüísticas-, las interpersonales individuales -automotivación y comportamiento ético-, y las interpersonales sociales -la comunicación interpersonal- encaminadas a fortalecer una ética de la responsabilidad (Verantwortungsethik).

\section{Propuesta Ignaciana de Educación y comunicación}

"La "física" como ciencia, natural moderna investiga solo determinadas relaciones del ente que llamamos

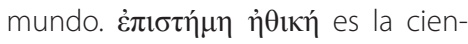
cia del $\tilde{\eta} \theta$ os, del comportamiento y la conducta del hombre hacia otros hombres y para consigo mismo: la ciencia del hombre. ... ahora el hombre es conocido en tanto que actúa para con los otros y para consigo mismo."

M. Heidegger. ${ }^{2}$

La comunicación constituye un eje transversal en los procesos de interacción humana; da cuenta de las elecciones que hemos realizado, la visión de mundo desde la cual actuamos -Weltanschauung particular que nos definey la posición que adoptamos frente a la comunidad. Desde nuestra especificidad como docentes de la Pontificia Universidad Católica del Ecuador, planteamos algunas consideraciones sobre la propuesta ignaciana de educación, su fundamento en la espiritualidad ignaciana y los lineamientos institucionales generales de la Compañía sobre los procesos comunicativos que caracterizan sus obras.

La Pontificia Universidad Católica del Ecuador está encomendada a la Compañía de Jesús, por lo tanto, asume la propuesta ignaciana de educación para desarrollar los procesos de aprendizaje-enseñanza; la propuesta ignaciana de educación tiene como fundamento los Ejercicios Espirituales que marcan la identidad de todas las obras confiadas a la Compañía de Jesús. Esta obra de san Ignacio de Loyola es esencial y desde su fundación propone un presupuesto dialogal: "el presupuesto radica en la voluntad de comunicación y la libertad de escucha" (Rui-Wamba, S.J.). El diálogo es una forma de interpensamiento y de aprendizaje social (cfr. Mercer, 2001), fundamental en los procesos de comunicación, más aún en la gestión de los procesos educativos; la Compañía ha establecido como principio esencial la interacción dialógica en el cumplimiento de sus procesos (Congregación General XXXIV), por esta razón, promueve un diálogo en cuatro ámbitos esenciales: el

${ }^{2}$ Heidegger, M. (2004). Lógica. La pregunta por la verdad. Madrid: Alianza Editorial, 12. 
diálogo de la vida, que implica un espíritu de apertura para compartir alegrías y penas, preocupaciones sobre los grandes problemas humanos; el diálogo de la acción, que se manifiesta en la colaboración para lograr un desarrollo integral y la libertad de las personas; el diálogo de la experiencia religiosa que permite compartir la riqueza espiritual de las tradiciones religiosas; y el diálogo del intercambio teológico que propende al entendimiento profundo y la valoración de las herencias religiosas de las personas.

La Compañía de Jesús se ha hecho cargo de las obras educativas como una manifestación de su apuesta por la educación. Desde su fundación ha optado por una propuesta educativa propia enmarcada en el ámbito del humanismo cristiano (Ratio Studiorum). En la época de su fundación no estuvo en total acuerdo con la propuesta que caracteriza a la educación escolástica, pues determinó que esta no conseguía relacionar el saber con una vida de virtud y servicio público (O' Malley, 1993, 259). Estos elementos han sido fundamentales para entender, fortalecer y gestionar las obras educativas confiadas a la Compañía desde sus inicios. Junto al humanismo, compartió la confianza en el poder formativo de la buena literatura -el lenguaje en su máxima expresión estética que cumple una función comunicativa sistémica-, lo que ha motivado que la propuesta ignaciana de educación haya fortalecido la importancia que se concede al conocimiento responsable y al cultivo del lenguaje, de la literatura y de la lectura crítica como elementos que fortalecen los procesos de aprendizaje. Polanco señalaba:

...el estudio de humanidad ayuda a la comprensión de la Escritura, es una propedéutica tradicional para la filosofía, ofrece una introducción pedagógicamente sana a otros temas, capacita a una persona para expresar mejor sus pensamientos, fomenta la pericia en la comunicación que exigen los ministerios de los jesuitas, y desarrolla la facilidad para diferentes idiomas que posee el carácter internacional de la Compañía. ( O'Malley, 1993, 261.)

En el desarrollo de las propuestas de evangelización de la Compañía, el Ministerio de la Palabra no se limitaba a la predicación, pues ampliaba su ámbito de incidencia comunicativa más allá de la lectio -instrucción o conferencia-y de la oratio -sermón u oración sagrada-: la Compañía proponía "escribir libros útiles para el bien común."3 La opción humanista cristiana en la propuesta educativa

\footnotetext{
${ }^{3}$ Es importante recordar que entre los primeros libros publicados en las imprentas de los jesuitas se encontraban los Epigramas de Marcial en 1558 (O'Malley, 1993, 146).
} 
de la Compañía, además de contribuir a la formación de un "carácter recto" que asuma la pietas como elemento esencial que se debía considerar en la conducta del estudiante y en su visión de las cosas, contaba con la esperanza de inculcar una piedad ilustrada - docta pietas- para la formación de buenos ciudadanos comprometidos con el bien común-, para quienes el saber y la cultura eran fines en sí mismos y por sí mismos, en un proyecto educativo que requiere de disciplina, perseverancia y diligencia. ${ }^{4}$

En este proceso, el lenguaje es asumido como un eje transversal, un espacio donde confluyen las diversas disciplinas y se integran en ámbitos de conocimiento que permiten una visión holística y sistémica del mundo; la propuesta ignaciana de educación realza la importancia del lenguaje para la autorreflexión y la reflexión necesarias para entender el mundo, para discernir -implicación del pensamiento y el lenguaje- y pasar a la trascendencia en la experiencia, en la puesta en práctica y en el testimonio de nuestras acciones desde el lenguaje: ...la educación jesuita está llamada a posibilitar procesos formativos donde cada estudiante construya conocimientos y realice acciones que promuevan su existencia humana y la del prójimo - vida, mundo, pluralidad-, pero también donde adquiera capacidades y experiencias que lo preparen y dispongan para el desarrollo de su vida espiritual -pensamiento, voluntad y juicio- (Racines, 2004, 7).

Los Ejercicios Espirituales de San Ignacio, asumidos como base para la propuesta ignaciana de educación, señala ya en la anotación 22 la importancia de "salvar la proposición del prójimo" (cfr. supra), lo que implicaría que el método del discurso comunicacional y educativo considera un proceso dialógico entre el ejercitante, -educador-, el ejercitador -educando- y Dios -la búsqueda de la verdad-, cuyo cumplimiento garantizaría el buen entendimiento y la buena aplicación de este método. En la misma dinámica, podemos concluir que la propuesta ignaciana de educación posee un presupuesto antropológico que estimula esencialmente este proceso dialógico:

\footnotetext{
${ }^{4}$ La propuesta de la Ratio Studendi de Benito Pereira para los procesos educativos iniciales de la Compañía establecía como meta de estudio el conocimiento de la verdad y la perfección de la mente humana. (cfr. Aristóteles), confería importancia a la formación de la mente y del carácter, al cultivo de los talentos propios, a la necesidad de prestar gran atención para cultivar los talentos espirituales del individuo y elevarlos hasta el punto más alto de perfección en el desarrollo de la inteligencia, la memoria y los juicios (O'Malley, 1993, 265).
} 
su objetivo fundamental es fortalecer la capacidad del diálogo con el otro -el prójimo-, desde el autoconocimiento, el ordenamiento de afecciones y el discernimiento para tomar decisiones que nos permitan realizar acciones que busquen y garanticen el bien común que se manifiesta en el respeto a la vida humana integral ${ }^{5}$.

La propuesta ignaciana de educación posee además un presupuesto de conciencia: su objetivo es conocer y elegir entre los pensamientos que se encuentran en cada uno de nosotros: "uno propio mío, el cual sale de mi mera libertad y querer, y otros dos, que vienen de fuera: el uno que viene del buen espíritu, y el otro del malo." (EE \# 32). Esta propuesta tiene como objetivo distinguir entre ilusión o realidad, nos sirve de advertencia contra el individualismo: el "yo simple", independiente de toda realidad no existe, la persona humana se desarrolla como persona con los demás y para los demás, en ella converge una razón dialógica ${ }^{6}$.

Las obras encomendadas a la Compañía poseen un principio y fundamento antropológico y teológico radical: el hombre es creado por y para Dios, en y con otras personas humanas de igual destino y dignidad, lo que permitiría que las personas humanas pudieran reconocerse a sí mismas desde la riqueza de la vocación y del amor auténtico. Esto es fundamental en la gestión de los procesos educativos y comunicacionales: no nacemos personas, nos hacemos personas; en esta afirmación está implícito un acto de voluntad y elección, ser personas humanas con las demás personas humanas nos ayuda en el proceso de conocimiento externo -aprehender, compartir e incidir en las circunstancias de la realidad en la que estamos insertos- y en el complejo proceso de autoconocimiento -aprehender, reconocer y gestionar con lucidez los diversos afectos, sentimientos y reflexiones-: el proceso de aprendizaje que al integrar mundo externo e interno conforman nuestras vidas y nos permiten interactuar con los demás.

La espiritualidad ignaciana impresa en la propuesta ignaciana de educación permite el reconocimiento de una identidad propia que consolida su gran riqueza y conforma su calidad específica: una tradición de larga data -s.XVI, la Ratio Studiorum- que conserva su pertinencia en la actualidad, un "evangelio de la persona" - prefigurado y elegido ya en los documentos fundacionales de la

\footnotetext{
${ }^{5}$ Hablar a otros y a sí mismo -recuerda Heidegger- es la conducta mediante la cual el hombre no sólo se hace notar como hombre, sino como guía y ese hablar dirige todas sus conductas: "al hablar de ello aclara, hace ver, define el modo de actuar y aquello de lo que hay que ocuparse" (M. Heidegger, 2004, 13).

${ }^{6}$ Cfr. Adela Cortina.
} 
Compañía- que permite establecer la importancia y la necesidad de discernimiento, el reflectir [sic] que nos relaciona con los demás y con nosotros mismos para iluminar nuestras intenciones, acciones y operaciones "puramente ordenadas en servicio y alabanza de su Divina Majestad" que implica permanecer fieles a la vida, y devenir persona humana con los demás y para los demás ${ }^{7}$. Esta decisión elegida desde el conocimiento de los valores y principios que consideran las convicciones profundas de las personas y determinan el modo de proceder de los individuos desde una reflexión sintiente y un sentimiento pensante que define nuestra condición de personas humanas integrales ${ }^{8}$.

Esta identidad tiene como características la responsabilidad y la espiritualidad comprometida, que se manifiesta en la coherencia entre el hablar y el accionar, por lo que el proceso educativo característico de la propuesta edu- cativa ignaciana constituye una filosofía del ser humano y su mundo: la identidad responsable propia y la identidad responsable de quienes han confiado en la propuesta ignaciana de educación: una educación inclusiva, que da paso a una espiritualidad incluyente que relaciona la fe con la justicia, con la cultura, con la ciencia; un proceso educativo y comunicativo que contribuirá a desarrollar una sabiduría espiritual como el medio para construir y consolidar realidades más justas, más humanas, más dignas a los ojos de Dios, cuyo objetivo fundamental es "ordenar los medios a los fines correspondientes".

Desde estas consideraciones, podemos señalar que la formación integral que propone la Pontificia Universidad Católica del Ecuador marca su identidad y, por ende, su calidad: está inserta dentro de los principios del humanismo cristiano e incorpora la pedagogía ignaciana, la vinculación de la propuesta

\footnotetext{
7 "... el pensamiento ignaciano, si bien contiene una descripción y ofrece una explicación en torno a la condición humana -al todo humano-, su finalidad no está en describir y explicar, sino en el provocar cambios, transformaciones estructurales, humanas y sociales, que se orienten y den cuenta de la presencia de Dios entre los hombres. En virtud de la naturaleza de esta finalidad, los enfoques sistémicos a ser utilizados en los procesos de comprensión y construcción del pensamiento ignaciano deben ser del tipo funcional-estructuralista (vg. Diseño de un cerebro de Ashby [1954]) y no del tipo 'estructural-funcionalista' (vg. Sistema social de T. Parson [1951)]. A fin de garantizar que las universidades ignacianas sepan cómo preguntarse a sí mismas, cómo examinarse de manera continua a sí mismas, acerca de la función que cumplen en las sociedades en que se encuentran insertas (Cf. Rodríguez, 1997) y, a través de ello -mediante la aplicación de modos semejantes a los que se consideran en los ejercicios espirituales ignacianos- adquirir capacidades para adecuarse a sus 'tiempos, lugares y personas'y para responder a las exigencias que se les presente." (Racines, F. "El Pensamiento Ignaciano y la Universidad Contemporánea”, 2016, pp.10-11).
}

${ }^{8}$ Cfr. infra, Giuseppina Grammatico. 
académica con la realidad social ecuatoriana, continental y mundial, la interdisciplinariedad en el desarrollo de pensamiento complejo, un diseño curricular flexible, la educación basada en competencias y resultados de aprendizaje para lograr un aprendizaje significativo que considera las necesidades de la persona del estudiante, el aprendizaje a lo largo de la vida, y la utilización responsable de las nuevas tecnologías y su incidencia en la sociedad.

Es importante señalar que esta identidad específica es plural y se desarrolla en una espiritualidad común con el objetivo de formar personas - proceso de formación integral, Bildung, no únicamente instrucción-que sean excelentes profesionales para que puedan transformar la sociedad, propender hacia un desarrollo integral individual y social, desde el compromiso, el discernimiento y el análisis de los grandes problemas sociales; personas que han desarrollado procesos responsables de aprendizaje que les permitan encontrar y ofrecer su experticia, su excelente preparación materializadas en las mejores soluciones, que ayuden a transformar los valores sociales, favorezcan el autoconocimiento y el conocimiento respetuoso del mundo, que puedan formar equipos integrados de laicos y jesuitas comprometidos con la vida y la trascendencia compartiendo metas comunes en el desarrollo conjunto de los proyectos emprendidos para alcanzar los fines propuestos.

Ya que la época en la que vivimos y actuamos está caracterizada por cambios constantes, ambigüedades, retos complejos, la comunicación también debe considerarlos para cumplir con coherencia su propuesta de servicio para alcanzar el bien común; la Compañía de Jesús ha considerado la comunicación como una "importante dimensión apostólica" de todos sus ministerios (cfr. Congregación General XXXIV, Decreto 15). ${ }^{9}$ Sus obras se proponen cumplir con las directrices del apostolado comuni-

${ }^{9}$ La Compañía cuenta con el Secretariado para la Comunicación Social (JESCOM), también ha incursionado en la sistematización de hitos distintivos en la formación y el desarrollo de la comunicación para la fe-justicia, pensando en mejorar los procesos de comunicación jesuita: 1970, SERPAL (Servicio Radiofónico para América Latina impulsado por Manuel Oliveira, S.J) ; el padre Pedro Arrupe, Prepósito General de la Compañía, aceptó la propuesta de Stephan Bamberg, S.J. para fundar JESCOM (Jesuit Communication) en Roma, el CSCC (Centre for Study of Communication and Culture), y el CICS (Centro Interdisciplinare sulla Communicazione Sociale) en la Gregoriana. La CPAL respondió a la solicitud de un plan de formación en comunicación social en reuniones cuyo fruto se encuentra en Disponer la vida para la misión (2009) y en la constitución del Proyecto comunicacional común. Se han consolidado la Red de radios, la Red digital SJ de América Latina y el Caribe, la Red de editoriales de AUSJAL, la Conferencias de redes EduTic y de los Homólogos de comunicación. Los SJR (Servicio Jesuita a Refugiados) tienen un Departamento de Comunicación; se ha establecido el Estudio de Video Roque Gonzáles en Santa Cruz, la Agencia de noticias Fides en Bolivia, el desarrollo del proyecto OPA (Oración Por el Arte), editoriales, y centros de audiovisua- 
cacional, todos estos proyectos permiten desarrollar el trabajo en redes como cuerpo apostólico, responden a las demandas de un entorno digital creciente, sin generalizar la superficialidad; no consideran audiencias, sino comunidades de personas donde existe la posibilidad de establecer contactos comunicacionales más transparentes y humanos desde la intersectorialidad, pues tienen como premisa el fortalecimiento y la difusión de la comunicación en todos los sectores: "La identidad de todo jesuita tiene una dimensión comunicacional. Todos somos comunicadores" (José Martínez de Toda, S.J., 2011).

\section{Lenguaje y responsabilidad en la comunicación}

"Hablar, no en el sentido reducido y remarcado de pronunciar un discurso, sino como hablar con otro, en y para el actuar y el obrar con otro. Este hablar con otro es un hablar con otro de circunstancias, oportunidades, medios, planes, tareas, relaciones, sucesos, destinos; es decir, en relación con lo anterior, hablar con otro del ser del mundo y del hombre. Este hablar con otro no siempre es actual, y sin embargo, el hablar sigue estando alli: en el repetir y el propagar, en el introduciry el presentar, en el hablar consigo mismo sin hablar en voz alta, en el responder ante si mismo: responsabilidad."

Martin Heidegger ${ }^{10}$

La Congregación General XXXV de la Compañía de Jesús confronta la cultura en la que estamos insertos en la actualidad, basada en el presente y en el énfasis en la consecución de autonomía, con la necesidad de construir un futuro solidario; el desarrollo tecnológico, tan avanzado en la ciencia y en los medios de comunicación, con una soledad que incrementa cada vez más las posibilidades de exclusión en la vida cotidiana de los seres humanos, dejando inalterada la amenaza a la integridad de la vida y del mundo (C.G. XXXV, 19).

En efecto, el desarrollo de la sociedad del conocimiento consigna altos estándares de calidad e insiste en la necesidad de su cumplimiento, entre ellos, señala los que conciernen a la educación como uno de los más importantes. Desde sus inicios, la propuesta ignaciana de educación abordó en forma minuciosa la enseñanza del lenguaje, debido a que su propósito era esencial: conocer la utilización óptima de las palabras para compartirlas con nuestros otros legítimos, pues las palabras nos conforman y abren la posibi-

les como el CEAFAX en Ecuador (José Martínez de Toda, S.J., 2011).

${ }^{10}$ Heidegger, M. (2004). Lógica. La pregunta por la verdad. Madrid: Alianza Editorial, 19. 
lidad de compartir y de compartirnos con los demás.

Afines a la propuesta ignaciana, concebimos la educación como un proceso de aprendizaje-enseñanza que debería considerar las necesidades sociales, que genere inquietudes, que busque capacitar a las personas para que gestionen el conocimiento, desarrollen los diversos pensamientos y concreten acciones desde el discernimiento -así se lograría la formación de personas responsables de sus acciones-, que se incorporen en procesos de interaprendizaje, interpensamiento (Mercer, 2001), innovación y en el aprendizaje social desde la utilización responsable del lenguaje y la comunicación que guíen la búsqueda, el conocimiento y la difusión de la verdad.

Este proceso exige planificaciones curriculares flexibles que permitan conocer y responder a realidades específicas, consideración de tiempos, lugares y personas; que favorezcan y ayuden en el desarrollo del trabajo en equipo, que incorporen conocimientos tecnológicos como herramientas válidas para gestionar el proceso de aprendizaje enseñanza: educadores que crean en su vocación profesión desde la responsabilidad y el compromiso social, propios de un espíritu integrador trascendente. Para gestionar este proceso en conjunto, hay que considerar al educador dentro de una nueva profesionalidad donde el cambio social sea visto como un sistema dinámico e impredecible - para el que debemos prepararnos como docentes y preparar a los estudiantes para saber responder y manejar la incertidumbre-, cambios que son propios de sociedades modernas con procesos de desarrollo complejos (S. I. Desafíos para nuestra misión hoy. CG XXXV). Para gestionar efectivamente este proceso, consideramos el desarrollo de competencias genéricas, cognitivas, lingüísticas, interpersonales, entre otras, que son necesarias para la formación integral de personas capaces de resolver los múltiples desafíos que nos plantea la realidad del mundo actual.

El Modelo de Innovación de la Educación Superior, MIES, (Villa y Poblete, 2005; Villa, 2011; Villa \& García, 2014) considera el proceso de aprendizaje del lenguaje como una competencia genérica instrumental, un eje transversal en la vida de todo ser humano, pues las competencias lingüísticas juegan un rol protagónico esencial en el desenvolvimiento evolutivo en las distintas etapas de los procesos metacognitivos, que tienen lugar en los procesos de aprendizaje.

El desarrollo de todas las competencias de pensamiento está favorecido por la utilización efectiva de las competencias lingüísticas; esto es fundamental para el cumplimiento de los procesos educativos, mucho más si consideramos la complejidad de la sociedad del cono- 
cimiento y la comunicación en la que estamos inmersos: "...cuantas más modalidades de pensamiento desarrolle una persona, mayores posibilidades intelectuales poseerá" (Villa y Poblete, 2005, 59).

La propuesta ignaciana de educación establece un proceso de formación para conseguir el desarrollo integral de personas reflexivas, analíticas, críticas, creativas, sensibles, propositivas, y el aprendizaje del lenguaje ocupa un papel capital en este proceso. Desafortunadamente, la realidad educativa que vivimos, en general, es otra: es fácil y común evidenciar grandes falencias en los distintos niveles de formación académica que revelan la proliferación de individuos que obedecen ciegamente un orden dominante, que no son capaces de cuestionar ni de cuestionarse, pues las palabras les resultan ajenas. Como sujetos sometidos a un proceso de banalización y de cambio vertiginoso, han devenido "otros", su aprendizaje se ha limitado a la repetición de normas que en muchas ocasiones resultan ininteligibles, por lo tanto, el lenguaje se sitúa en el ámbito de los datos descartables:

...les resulta difícil apropiarse de las palabras para proponer soluciones, mirarse en el otro, trabajar por sí mismos y por los demás para conseguir sociedades justas, equitativas y dialógicas donde interactuemos como personas. Estas complejidades dejan al descubierto que asistimos -algunos como protagonistas, otros como testigos impotentes- a un proceso de fragmentación social, como espectáculo propio de una sociedad líquida, como diría Zygmunt Bauman (2013), una sociedad que plantea como meta "vivir el momento", liberarse de responsabilidades, rechazar el compromiso; que promueve el temor de detenerse a pensar, a sentir, a establecer "modo y orden" en decisiones y acciones que se expresan con palabras o con la ausencia de ellas en una terrible espiral de silencio. Estos elementos se evidencian en la cotidianidad devaluadora del lenguaje y su accionar en las aulas, en su manera de ver, interpretar y actuar en el mundo (Merchán y Zary, 2016, 168).

Consideramos que la banalización es una característica propia de la modernidad líquida, este problema es un reto que debemos asumir como comunidad para resolverlo en forma creativa e innovadora desde la palabra, desde el conocimiento y la utilización responsable del lenguaje como invalorable facultad que nos permite expresar cómo aprehendemos el mundo, cómo lo asimilamos y lo vivimos en las diferentes situaciones comunicativas, en la coti- 
dianidad, en lo académico, en lo íntimo de nuestro ser.

El desarrollo de nuestras competencias comunicacionales exige conocer las palabras, respetarlas, sentirlas nuestras, utilizarlas para compartirlas con los demás y fortalecer la lectura de la realidad, combatir la escasez -o ausencia- de puntos de orientación estables, la inaccesibilidad a una estructura sistémica, la transitoriedad, fragilidad y vulnerabilidad de los vínculos, la libertad como problema, la incapacidad de tomar decisiones en forma consciente y responsable (Bauman, 2005; Reker \& Woo, 2011).

La didáctica tradicional que aún está presente en las aulas de primaria, secundaria y en el nivel académico superior -la mayor parte de las veces- no ha considerado estas necesidades ni ha permitido el cabal desarrollo del lenguaje ni su relación con la comprensión del mundo, la aprehensión responsable de la realidad, ni con las diversas situaciones comunicativas en las que participamos cotidianamente como integrantes de comunidades comunicativas.

Debemos recordar que el lenguaje cobra una presencia fundamental en todos los procesos de comunicación: en las actividades académicas, en el autoconocimiento íntimo de nuestro ser, en la palabra que musita nuestros nombres y nos congrega, que nos hace sentir amados, que nos incorpora en la comunidad, que reivindica nuestra memoria originaria, que clarifica nuestros propósitos y objetivos, que hace factible que compartamos con los demás nuestras creencias, nuestras inquietudes, nuestras opciones vitales, que nos ayuda en el proceso de interpretación de las palabras de los demás y con los demás:

Lograr expresar y compartir toda esta experiencia de pensamiento, sentimiento, de pensamiento sentiente y de sentimiento pensante, como afirmara Giuseppina Grammatico (2008), gestionar estos pensamientos a través de la palabra, es una tarea -entre las más importantes- que debemos cumplir en el proceso de aprendizaje-enseñanza de la lengua (Merchán y Zary, 2016, 169).

Las iniciativas que en el mundo se han generado sobre los procesos para conseguir el fortalecimiento de las competencias lingüísticas revelan la profunda preeminencia del tema: la necesidad del trabajo sostenido en el conocimiento, valoración, respeto, amor y apropiación manifiestos en el uso adecuado del lenguaje y la riqueza que pone a nuestra disposición, el aprendizaje del lenguaje gestionado como un proceso interdisciplinario, como un referente para la comunidad académica y una necesidad en el desarrollo de líneas de investiga- 
ción que guíen el trabajo docente hacia la búsqueda de las mejores estrategias para el proceso de aprendizaje-enseñanza del lenguaje.

La propuesta ignaciana de educación propende hacia la consecución de la autonomía del estudiante (Velasco, 2014; Liévanos-Álvarez, 2016), lo que implica también asumir su responsabilidad en la comunidad. "1 Esta autonomía implica la consideración del prójimo, las oportunidades de comunicación, interpensamiento y aprendizaje social, un próximo -un otro- que sirva de magnitud, de referencia central para la construcción de nuestra identidad en la realidad que nos remite a la comunidad, donde ponemos en práctica un "imperativo estético... Si quieres conocer, aprende a actuar", y "un imperativo ético... : actúa siempre de modo que se incremente el número de elecciones". De esta manera, las personas podemos construir a partir de un actuar (wirken), actuando conjuntamente, nuestra realidad (Wirklichkeit) (Von Foerster, 2010, 55).

\section{RESULTADOS}

\section{Leer y escribir como fundamento de una comunicación humanizadora}

La ética racional de la comunicación tiene un carácter universal por cuanto aspira al bien común compatible con los intereses de todos los miembros de la comunidad. Su aplicabilidad radica en la posibilidad de mediación "discursiva" entre las máximas individuales y las normas éticas colectivas. De ahí que aprender a comunicarnos de una forma eficiente se vuelva una necesidad vital para afrontar los desafíos que la vida en la sociedad conlleva. Se necesita una nueva forma de diálogo que genere una conversación que una a las personas desde su creativi- dad y diversidad cultural (Francisco, 2015). La sociedad tiene una deuda pendiente con la gestión sostenible de los recursos y el respeto de los derechos de las personas. La formación en la responsabilidad, fundamentada en una relación intersubjetiva, se vuelve una tarea ineludible a fin de establecer acuerdos o consensos que no dependan de la voluntad individual de los poderosos, sino de la consideración de la propia trascendencia del ser humano y el universo. El "Buen Vivir" o el anhelo de una vida buena, como elemento filosófico trascendental, es una propuesta válida para las generaciones pasadas, presentes

\footnotetext{
${ }^{11}$ Este principio se marca como un eje transversal que va desde las propuestas humanistas clásicas hasta las propuestas actuales, entre ellas, la del construccionismo radical (cfr. Heinz von Foerster, 2010).
} 
y futuras, ya que hace honor a la vida y a la libertad. Su pretensión es alcanzar una independencia ideológica que posibilite la búsqueda intersubjetiva de la verdad, a modo de una ética científica con normas éticas universales (Lellouche, 2001) por cuanto la comunidad investigativa es también de carácter infinito, al igual que sus argumentos. Las necesidades de la humanidad son de interés de la comunidad de comunicación cuyo lenguaje performativo entra en diálogo desde el horizonte de una ética de la responsabilidad y la libertad.

Sin embargo, a pesar del fundamento y desarrollo de una ética de la comunicación para la verdad y transparencia, esto no es suficiente para hablar de humanidad. El acto de leer y escribir, hoy logros indiscutibles de la sociedad en lucha contra el analfabetismo, es un proceso de interpretación de la realidad, y por ende, una comunicación humanizadora de la misma. Todo el proceso de adquisición del lenguaje oral y escrito apunta a un desarrollo progresivo de la forma, el significado y la función (Colomer, 1997). En este proceso se interrelacionan al menos tres factores en la enseñanza-aprendizaje de la lectura: la persona que lee, el texto y el contexto de la lectura. La armonía existente entre estas variables favorecerá u obstaculizará el desarrollo de las competencias involucradas en el proceso lector, lo que conlleva un mayor o menor grado de goce estético, conocimiento y comprensión del mundo y, finalmente, de humanidad.

De lo mencionado anteriormente se espera el fortalecimiento de la comunicación para la responsabilidad. Es notable que en el proceso de comprensión de un texto interactúan tanto las estructuras cognitivas como afectivas de la persona. De ahí que en la actitud del lector se evidencien su empuje o el temor al fracaso, así como la elaboración posterior al acto de lectura mediante inferencias y razonamientos que favorecen el proceso de metacomprensión. En conclusión, una ética de la comunicación no se puede improvisar, sino que se nutre del uso de estrategias inconscientes y del razonamiento crítico proporcionado por el proceso de lecto-escritura que adquiere una persona. Este proceso de humanización es la base para tomar buenas decisiones en su vida y con la mira hacia la dimensión social del bien común.

\section{La perspectiva ignaciana para una comunicación comprometida con el bien común: el presupuesto para salvar la proposición del prójimo como un instrumento del discernimiento}

La propuesta ignaciana de educación consolidada en el Paradigma Pedagógico Ignaciano es el fruto de una 
tradición metodológica que brota de los Ejercicios Espirituales para vencer a sí mismo y ordenar su vida, sin determinarse por affección [sic] alguna que desordenada sea (EE \# 21). Su praxis educativa está orientada a la formación integral de la persona. Aquí se recoge la formación humana en virtud y letras, acuñada por la Ratio Studiorum, documento que rigió la formación de la Compañía de Jesús durante algunos siglos. La pedagogía ignaciana cuenta con un método ampliamente reconocido -si bien a veces desestimado- a través del orden y método de la contextualización, experiencia, reflexión, acción y evaluación. A través de la integración de estos elementos (contexto, experiencia, reflexión, acción, evaluación) que fluyen concatenados entre sí, se favorece una serie de procesos acompañados y ordenados hacia la excelencia académica y humana.

En el centro de este proceso se encuentra la persona de cada uno de los miembros de la comunidad educativa. Su fin es la búsqueda del horizonte de libertad para el crecimiento y maduración en todas las dimensiones humanas (ética, espiritual, cognitiva, afectiva, comu- nicativa, estética y sociopolítica). El método aprehendido en este proceso de discernimiento no está exento de desafíos tanto locales como globales, sin embargo es la herramienta válida para ser colaboradores de una misión'12. En pocas palabras, una propuesta para el ordenamiento de un compromiso ético libre y responsable en la transformación ${ }^{13}$ de la sociedad y el mundo.

El rendimiento académico no puede divorciarse de la formación humana en excelencia (magis), puesto que ambos elementos en combinación son los que construyen la identidad personal y la formación integral de la persona (Ruiz, 2014). En el caso de la vida de los jóvenes que educamos, contemplamos la vibración de la juventud que busca una vida mejor, el gozo de muchos ante la belleza de la creación y las múltiples formas en las que muchos ponen sus propias cualidades al servicio de los demás (CG XXXVI, 1.1) ${ }^{14}$.

A fin de profundizar en una propuesta de formación ética en la responsabilidad es imprescindible conocer más de cerca a la persona a la que se pretende formar, en este caso los jóvenes que ingresan a la universidad. ¿Cómo respon-

\footnotetext{
${ }^{12}$ La colaboración con otros está en el corazón de la misión. Cfr. Decreto 6, La colaboración en el corazón de la misión, No. 3, 15, 30, Decretos de la Congregación General XXXV de la Compañía de Jesús.

${ }^{13}$ Cfr. Herman Castelino, S.J., "Educación para la transformación. Un colegio jesuita en el siglo XXI". Conferencia Jesuita de Asia Meridional, Marzo 2005.

${ }^{14} \mathrm{Cfr}$. Decreto 1, Compañeros en una misión de Reconciliación y de Justicia, No. 1, Decretos de la Congregación General XXXVI de la Compañía de Jesús (2016).
} 
de el joven de hoy a las preguntas centrales de la identidad y el sentido de la vida? ¿Quién soy yo? ¿Puedo amar y ser amado? Al parecer hay una búsqueda de sentido, pero desde otros referentes diversos a los de generaciones anteriores. El mundo virtual que los envuelve les proporciona visiones fragmentadas de la realidad. La tendencia audiovisual marca con fuerza sus gustos y preferencias, lo que deriva en el imperio de la moda, del sentirse conectado a un fenómeno global en forma simultánea ${ }^{15}$.

Sin pretender una generalización absoluta sobre la persona de los jóvenes, es posible observar un colectivo de personas alegres, creativas y dinámicas. La gran paradoja es que a pesar de sentirse parte de un grupo, el narcisismo de una sociedad individualista troca su juventud en gran fragilidad para su compromiso y toma de decisiones, probablemente por el temor al fracaso y lo incierto de su futuro. Una respuesta posible es la evasión mediante el consumo de alcohol y cualquier actividad que genere adrenalina y adicción (telefonía celular, internet, televisión). De otra parte el desencanto aludido anteriormente conlleva descon- fianza y sospecha de los grandes relatos e instituciones. Ante la autoridad buscan la independencia con mucho temor a la responsabilidad (Man-Ging, Merchán, Racines, 2010).

La espiritualidad ignaciana que fundamenta el proceso de aprender a aprender mediante una visión dinámica y positiva del ser humano, pretende ofrecer un camino de integración a través de la formación en una ética responsable. Del desorden de los afectos se busca la transformación hacia el Origen que le origina y al fin que le plenifica. ${ }^{16}$ Para alcanzar una vida buena se propone como punto de partida el presupuesto de los Ejercicios (EE \# 23) para buscar puntos de acuerdo y de sintonía con la proposición del prójimo, es decir, basado en la confianza y la búsqueda de un bien mayor. Dicho comienzo o principio que regirá la nueva manera de comunicarse implica el paso transformador de una existencia autocentrada y/o cerrada a una nueva libertad de la vida abierta por la alteridad y solidaridad de la interdependencia, y en definitiva por una actitud contemplativa desde el amor.

\footnotetext{
${ }^{15}$ Es la generación del "orgasmo inconcluso" cuya satisfacción no se logra nunca por el zapping de pasiones (continuo, frenético y vertiginoso) al que es sometida. Cfr. Juan Cristóbal Beytía, S.J. "La cultura juvenil y sus desafíos" en Revista de Espiritualidad Ignaciana, XXXIX. 1/2008. No. 117, 11-25.

${ }^{16}$ Diversas disciplinas científicas insisten en el proceso de transformación del ser humano. Cfr. J. Melloni. "La espiritualidad ignaciana como proceso de transformación" en Revista Manresa vol. 81 (2009), Madrid, Centro Loyola, 363-377.
} 
La Pedagogía Ignaciana demanda entonces creatividad para sustituir una concepción ampliamente difundida en nuestro medio como es la del poder para usufructuar, trocándola en una consecuencia más evangélica como el poder para servir. Un servicio abierto a los valores que fundamentan los cuatro pilares de su estructura y dinámica interna: utilitas, iustitia, humanitas y fides. Se trata por ende de un servicio que está en la base del humanismo cristiano contemporáneo para iluminar la actitud responsable con la sociedad y con las futuras generaciones de nuevas personas para los demás, agentes de cambio sustancial.

El rol del maestro en este proceso de aprendizaje-enseñanza-aprendizaje es no menos importante que la actitud de magnanimidad que se requiere en el estudiante. Se espera un perfil docente basado en el respeto y la paciencia frente al proceso educativo. Solo así se toma en cuenta la dignidad y libertad de los actores del acto educativo. De igual forma, se espera un docente-investigador riguroso; pero además comprensivo y reflexivo que no manipula a los estudiantes que se le han confiado, sino que más bien los entrena en el conocimiento de la realidad con espíritu crítico.

\section{Hacia una ética de la comunicación: ¿un poder para servir?}

"Así pues, para resolver el problema de una ética posconvencional de la responsabilidad, sólo parece quedar el camino de la ética discursiva; es decir, la cooperación solidaria de los individuos ya en la fundamentación de las normas morales y jurídicas susceptibles de consenso, tal como es posible, principalmente, por medio del discurso argumentativo."

Karl-Otto Apel ${ }^{17}$

Dentro de la complejidad que caracteriza a nuestra época, es fundamental recordar que los procesos educativos incluyen un nivel axiológico; los valores responden a necesidades, son estas las que confieren la propiedad que adquiere para alguien un objeto o una actitud. Se ha definido la ética como una ciencia práctica relacionada con las acciones de la persona, controladas por la razón y por la voluntad. En la carrera de comunicación, es importante considerar que los valores comprometen acciones futuras, el desarrollo efectivo del lenguaje para conocer y difundir la verdad es fundamental ${ }^{18}$ : el ser veraz se convierte así en

${ }^{17}$ Apel, K.-O. (1991). Teoría de la verdad y ética del discurso. Barcelona, Paidós, 148-149.

${ }^{18}$ La ética en occidente, inmersa en la investigación filosófica, tiene una tradición de casi veinticinco siglos. Ya Aristóteles recordaba en la Ética a Nicómaco, que en todo lo que hacemos nos preguntamos por el 
un "deber ser" desde la decisión libre del sujeto (Herrán y Restrepo, 2005).

Dentro del desarrollo de la ética y sus diferentes corrientes y propuestas ${ }^{19}$, hemos decidido detenernos en la Ética comunicativa propuesta por Karl-Otto Apel y J. Habermas; su propuesta de una "ética dialógica de la responsabilidad solidaria" plantea buscar valores comunes gracias al diálogo y fortalecer la moral civil, pues "sin importar cuáles son las creencias últimas de las personas, obligan a colaborar en el perfeccionamiento de los grupos sociales a los que pertenecemos." (L. J. González Álvarez, citado por Herrán y Restrepo, 2005, 28). Este concepto de responsabilidad social implica integralmente al comunicador, lo define como sujeto ético.

Mantener la coherencia con nuestros principios éticos marca nuestro sentido de autonomía responsable; Adela Cortina nos previene contra el "politeísmo moral", donde coexisten varias morales en el obrar cotidiano de un individuo, de lo que resultan acciones incoherentes; en la era de la comunicación y el conocimiento necesitamos una ética dinámica, práctica, dialogante y mínima, que nos permita analizar los contextos y sus consecuencias, que posibilite dar prioridad a ciertos principios: la ética de los comunicadores no puede ser una ética de códigos exclusivamente.

En un sistema ético, los principios, los valores y las virtudes se relacionan entre sí; en el ámbito de la comunicación se podría señalar como principios fundamentales: veracidad, autenticidad, búsqueda del bien común, solidaridad, justicia, libertad, responsabilidad; estos principios se conjugan con valores como la verdad, sinceridad, equidad e independencia; principios y valores se relacionan con las virtudes del ser humano: franqueza, altruismo, compasión, autonomía, fortaleza, valentía, prudencia. (Herrán y Restrepo, 2005).

Los códigos éticos consideran para su codificación las actitudes y tradiciones permanentes fundadas en la naturaleza humana. La ética surge de la toma de conciencia de los individuos de su propia naturaleza o la naturaleza de las actividades que realizan, sus códigos nacen del buen sentido, constituyen la expresión de su conciencia y no necesitan de coacción externa; consideran como un deber investigar la verdad, plantean la necesidad de conjugar lo

fin en virtud del cual realizamos nuestras acciones.

${ }^{19}$ Entre ellas, el Escepticismo ético, que considera la ética como asunto individual; el Voluntarismo fundamentado en la superación y el deber; la Ética de la satisfacción sostenida por los utilitaristas; la Ética de bienes que establece que lo bueno corresponde a lo más profundo de los seres humanos; la Ética de los valores que plantea que el sentido del valor incide en nuestra forma de percibir la perfección de las cosas. 
ético y lo técnico. De acuerdo a los principios éticos, es indispensable dar prioridad al bien común sobre los intereses comerciales: los comunicadores asumen el objetivo social como parte integrante de la técnica de su profesión. (Herrán y Restrepo, 2005).

La ética de los comunicadores está construida sobre dos bases fundamentales: el amor y el respeto por la verdad, y el servicio del bien común. Esto podemos encontrarlo suscrito en el Código de la UNESCO: "En el periodismo, la información se comprende como un bien social y no como un simple producto" (Herrán y Restrepo, 2005, 40 ). La información como bien social está determinada por las conveniencias y necesidades del bien común.

En esta tónica, recordemos que el Código de la Comunidad Europea de Periodistas establece: "Toda acción periodística debe estar dirigida al bien espiritual social, intelectual y moral de la comunidad. El periodismo debe ser un servicio de intereses colectivos, con funciones eminentemente sociales dirigidas al desarrollo integral del individuo y de la sociedad" (Herrán y Restrepo, 2005, 40.), lo que coincide con las propuestas de la
Gaudium et spes. El bien común constituye el bienestar de la sociedad como totalidad; por esto, los intereses particulares quedan subordinados a ese objetivo.

Las consecuencias tecnológicas de la ciencia en la actualidad confieren a las actividades humanas un alcance y una amplitud que nunca antes habían tenido. Una cuestión de responsabilidad en el nivel de los intereses comunes vitales de la humanidad es el problema del bien común como praxis común auténtica, es la causa de la crisis de la humanidad contemporánea, por lo que no se necesita únicamente una ética de la convicción Gessinungsethik, sino una ética de la responsabilidad Verantwortungsethik. (cfr. Max Weber, citado por Lellouch, 2001, 202.)

Al revisar la propuesta de Karl-Otto Apel ${ }^{20}$ sobre la ética del discurso, encontramos que él debate sobre los límites de esta ética desde una manera nueva: en forma comunicacional y discursiva; el principio fundamental de una ética racional de la comunicación radica en el principio de universalización enraizado en los supuestos necesarios de todo discurso argumentativo. Este autor define la comunidad de la comunicación como

\footnotetext{
20 Justificamos nuestra selección porque la ética racional está considerada como ética del discurso, lo que nos permite relacionar los temas que hemos desarrollado y cuya conjunción es fundamental para las consideraciones sobre una ética de la comunicación en una obra educativa confiada a la Compañía de Jesús: la propuesta ignaciana de educación, las acciones comunicativas de la Compañía, el desarrollo de las competencias lingüísticas en el conocimiento, la búsqueda, el conocimiento y la difusión de la verdad.
} 
necesitada de normas formales de una situación ideal de habla, en la que prima la simetría y el derecho equitativo a hablar, que considera el principio teleológico de la redención de las pretensiones e intereses de todos los participantes virtuales de una discusión argumentativa.

Las características más sobresalientes de esta propuesta corresponden a las condiciones que debe cumplir este principio: constituirse en una "metanorma" para suministrar un fundamento sólido a toda norma concreta válida, poseer la calidad de valor universal, que supera el relativismo moral y que debe ser aplicable, "...la unilateralización técnica de la racionalidad se sitúa, según Apel, dentro de una estructura antropológica" (Lellouch, 2001, 208).

Las normas éticas que están implícitas en la justificación del sentido en la comunidad de comunicación son: la sinceridad -imposibilidad de la mentira continuada-, el principio de caridad interpretativa -esfuerzo de comprensión del otro-, y el examen imparcial de los argumentos -igualdad de las partes de la comunicación-. La condición pragmática del lenguaje incorpora la intencionalidad comunicacional, la referencia implícita al otro se debe considerar como elemento que da origen a todo acto constitutivo de sentido, y está supuesta en toda comprensión, auto-comprensión, y por supuesto, en la intercomprensión dentro de la comunidad comunicacional.

La ética apeliana presenta así su principio fundamental: "Todas las necesidades humanas, como pretensiones virtuales que son, deben ser objeto de la preocupación de la comunidad de comunicación con todos los demás, por la vía argumentativa" (Lellouch, 2001, 230). Se fija entonces el principio de una responsabilidad que vincula solidariamente a cada miembro de la comunidad de comunicación con todos los demás. Apel vislumbra aquí la función irreemplazable de la libertad de la voluntad y del "acto existencial" en el reconocimiento o la negación de las condiciones normativas de posibilidad de la autocomprensión humana (Lellouch, 2001). La ética comunicacional se concreta así en principios reguladores de una estrategia moral doble: la supervivencia de la comunidad comunicacional real y la realización histórica de la comunidad de comunicación ideal.

Las reflexiones que se han desarrollado sobre la ética comunicacional se conjugan con la propuesta ignaciana de educación para propender una participación responsable, crítica, respetuosa y deliberante. Se aspira a que esta propuesta educativa respete el pensamiento divergente y propenda al desarrollo integral, que invite a la acción para relacionarse en forma abierta, comprometida, trascendente y responsable con la 
comunidad comunicacional y sus necesidades específicas. La propuesta ignaciana de educación privilegia la gestión del método, prioriza el diálogo y la visión hologramática de la realidad desde la creatividad, el ingenio y la innovación social. Estos elementos son parte de un proceso de aprendizaje en un contexto que toma en cuenta las dimensiones ética, estética, social y espiritual -la realidad toda- para servir mejor.

\section{DISCUSIÓN \\ GLOBALIZACIÓN Y RESPONSABILIDAD EN LA ÉTICA DE LA COMUNICACIÓN}

El proceso de globalización que experimentamos desde hace algunos años plantea una complejidad social que produce una nueva economía informacional/global y la cultura de la virtualidad real. La lógica inserta en esta economía, en esta sociedad y en esta cultura subyace en la acción social y las instituciones de un mundo interdependiente. De acuerdo a Manuel Castells (2001), el nuevo orden social y económico en el que estamos inmersos constituye la sociedad de la información y se caracteriza por estar enraizada y dirigida por el desarrollo, la expansión y la circulación de una información y entretenimientos digitales basados en la informática, que son electrónicos y globalizados. Su fuente de productividad radica en la generación de tecnología del conocimiento, proceso de la información y comunicación simbólica que produzca, valga la redundancia, la acción del conocimiento en el propio conocimiento como principal fuente de productividad en un círculo virtuoso de interacción.

Como consecuencia de esto, vivimos la sociedad del espectáculo que propicia la banalización. Este proceso caracteriza a una sociedad líquida, y requiere una propuesta alternativa a fin de no quedarnos "atrapados en el pensamiento único asumido como el paradigma de lo aceptable y deseable, que como todo paradigma condiciona todo modo de pensar y de actuar." (Ander-Egg, 2005, 162.)

Estamos convencidos de que la propuesta ignaciana de educación es la propuesta alternativa que nos ayudará a dar "modo y orden" en la formación de los actantes de los procesos de aprendizaje dentro de un proceso educativo crítico, responsable, humanista y trascendente que promueve la comunicación (Lúquez, Fernández \& Bustos, 2015; 
Navarro, 2015). Una universidad encargada a la Compañía de Jesús, con responsabilidad social universitaria clara, habrá de considerar dentro de su dinámica que la desigualdad vulnera la dignidad humana; que la falta de oportunidades de desarrollo deteriora la calidad de vida de las personas; que la universidad, como un actor social fundamental y estratégico, cuenta con experiencia, genera conocimiento, está integrada por personas que demuestran valentía en la defensa de los ideales, esperanza en la posibilidad de un cambio y generosidad para realizar un trabajo comprometido en la gestión de acciones para la consecución de igualdad, equidad y justicia social. Desde la responsabilidad social universitaria, se busca la reconexión de la universidad con el contexto social en la que está inserta y se la anima a reencontrar su identidad para discernir sobre cómo puede responder la universidad a las necesidades de la sociedad desde su misión que contempla la comunicación como un eje transversal de sus acciones.

De acuerdo a la Congregación General XXXIV, la comunicación debe desarrollarse en forma comprometida y responsable en las obras encargadas a la Compañía, esto implica también las obras del ministerio académico, para conseguir:
- Respuestas al "giro cultural" que caracteriza la era de la "revolución informativa", a los retos de una "cultura no lineal" que se orienta a la imagen, que enfatiza la intuición y los afectos para interpretar el mundo.

- Utilización democrática de los medios de comunicación y su lenguaje con fines positivos y que valoren la trascendencia, que propugnen el crecimiento integral de la persona humana, la formación de usuarios y profesionales críticos de la comunicación social.

- Conocimiento del lenguaje y símbolos, la fuerza y las debilidades de la cultura de la comunicación moderna.

- Utilización del nuevo ambiente de la comunicación como medio para establecer contactos con las personas, promoción de la alfabetización, la instrucción, la solidaridad.

- Promoción de la fe y la justicia en una acción coordinada e interdisciplinaria, que permita un flujo equitativo de información y el conocimiento responsable de la ética de la comunicación.

- Educación y uso creativo del lenguaje y las técnicas de comunicación como participantes del diálogo social.

- Comprensión crítica de la realidad para evitar la manipulación y domi- 
nación de los medios.

- Utilización creativa de medios, incorporación de medios alternativos para lograr una comunicación más directa con el pueblo.

- Aseguramiento del conocimiento crítico de la retórica de la nueva cultura de la información y el fomento de su dimensión estética.

- Desarrollo de aptitudes que promuevan el trabajo en equipo.

- Aprendizaje efectivo del uso de los medios y las tecnologías de la información en el apostolado.

Considerando estos señalamientos, la propuesta ignaciana de educación plantea que la respuesta a las necesidades sociales desde el enfoque tecnológico debe integrarse con una función instrumental que sirva para la sistematización de los procesos que caracterizan la propuesta de una educación integral donde las nuevas tecnologías de la información y la comunicación se utilicen y se desarrollen como TACs -Tecnologías de Aprendizaje y Conocimiento-, y como TEPs -Tecnologías de Empoderamiento y Participación-, puestas al servicio del aprendizaje social (Reig, 2012) que permitirían gestionar procesos efectivos de aprendizaje con estudiantes en ámbitos de "la realidad aumentada" ${ }^{21}$ que conlleva la consideración de los contextos de "la sociedad aumentada", esencialmente una sociedad de participación en la era de la "hiperconectividad22; la utilización de estos instrumentos tecnológicos aseguran los entornos personales de aprendizaje y la interacción en la comunicación dentro de una sociedad que asume el diálogo como elemento esencial para realizar el paso decisivo de la interacción a la participación y el empoderamiento, lo que permitiría cumplir con el principio de"tanto cuanto"23.

La propuesta ignaciana de educación contempla en el ámbito de un modelo sociocrítico y humanista cristiano la ejecución de procesos educativos que propendan a dotar a los agentes del proceso de aprendizaje-enseñanza -educadores y educandos- de las competencias, los logros de aprendizaje y los instrumentos necesarios para que consigan:

\footnotetext{
${ }^{21}$ Cfr. Dolors Reig, videoconferencia: Realidad aumentada y aprendizaje. (2012).

${ }^{22}$ Cfr. Dolors Reig. (2013) Los jóvenes en la era de la hiperconectividad.

${ }^{23}$ El principio que rige los procesos de comunicación y la utilización de los medios en las prácticas comunicativas de la Compañía de Jesús, "Sé tú quien cuente tu historia", se relaciona con la importancia de los trabajos en los medios para crear contenidos; este principio está en uso desde hace algunas décadas y se ha aplicado en la cobertura de la CG 36. Cfr. CPAL. (2016). La importancia de la Narrativa: La comunicación en la CG36.
} 
- Pensar y preparar su porvenir sin olvidar que pertenecen a pueblos con tradiciones específicas que están insertos en los procesos de globalización y mundialización, y que deben asumir los retos dentro de la complejidad que éstas plantean - pertinencia de la competencia de pertenencia histórica-.

- Diversificar las trayectorias, encontrar soluciones alternativas, innovadoras, y desarrollar las competencias lingüísticas que se interrelacionan con el pensamiento analítico, pensamiento crítico y autocrítico, pensamiento sistémico y el pensamiento divergente para discernir sobre las mejores soluciones que permitan conseguir un mundo más humano, equilibrado, justo, equitativo y solidario.

- Mantener abiertas las perspectivas que conducen a la innovación y no conformarse con aceptar propuestas desacreditadas que han sido presentadas por el sistema establecido como la panacea para que el mundo siga en marcha -desarrollo de la competencia de pensamiento crítico y pensamiento divergente-.

- Reflexionar sobre los errores pasados, presentes y futuros a fin de repararlos y superarlos; asumir con humildad, lucidez y ética nuestras limitaciones y las respuestas que se han generado en función del servicio que responde a otros intereses que no han considerado a toda la humanidad, especialmente a los estamentos más frágiles de la sociedad-desarrollo de competencias de pensamiento autocrítico, de liderazgo social, de comunicación, de sentido ético-.

\section{CONCLUSIONES}

Concluimos entonces que el objetivo de la propuesta ignaciana de educación es conducir a una transformación radical en la forma de comunicar que implica pensar, actuar, sentir, entender la vida desde la formación de hombres y mujeres conscientes, críticos, compasivos, competentes, que buscan el mayor bien en el compromiso de la fe y la justicia para mejorar la calidad de vida de los seres humanos, especialmente de los pobres de Dios, los oprimidos y abandonados.

Los planteamientos de la propuesta ignaciana de educación son fieles a una propuesta humanista cristiana que garantice la formación holística de la persona humana; considera la persona 
del estudiante como el centro de atención preferente del proceso aprendizaje-enseñanza.

La educación ignaciana se preocupa por la formación de personas integrales, consciente de sus fortalezas y posibilidades de cambio; personas autónomas que reconocen la importancia de la trascendencia y, por lo tanto, la responsabilidad de incidir con sus acciones sobre su realidad y pueden testimoniarla efectivamente mediante el desarrollo de competencias lingüísticas orales y escritas -con coherencia ética y estética- que favorecen la comunicación responsable en comunidades comunicacionales paritarias.

La propuesta ignaciana de educación considera el derecho a la trascendencia en el proceso de formación de la persona humana, desde un cuidado prolijo, que pone énfasis en el desarrollo de la reflexión, la criticidad, los principios éticos, la utilización responsable del lenguaje y la comunicación dentro de una propuesta inserta en los modelos socio-crítico y hermenéutico para asegurar una comunidad comunicacional respetuosa de los principios, valores y actitudes que caracterizan la búsqueda y la difusión responsable de la verdad.
La propuesta ignaciana de educación considera importante el enfoque tecnológico, evita la generalización de la superficialidad y reconoce una función instrumental a las TICs: se plantea su gestión responsable y efectiva como TACs y TEPs al servicio del aprendizaje social y del desarrollo de comunidades comunicacionales.

La Compañía de Jesús posee planteamientos claros sobre la importancia de la capacitación y difusión de la comunicación comprometida y responsable en las obras encargadas a ella; considera el contexto cultural de la era de la "revolución informativa"; trata de responder a los retos de una "cultura no lineal"; propende la utilización democrática y crítica de los medios de comunicación y su lenguaje con fines positivos; procura establecer vínculos solidarios con las personas para que participen del diálogo social desde la utilización creativa del lenguaje y el conocimiento responsable de la ética de la comunicación.

La ética comunicacional no está vacía, implica la consideración de una comunidad que se establece en función del diálogo paritario en el proceso de argumentación, de intercomunicación, de la gestión de competencias que aseguren la comprensión, la autocomprensión y la intercomprensión. 


\section{BIBLIOGRAFÎA}

Ander-Egg, E. (2005). Debates y propuestas sobre la problemática educativa. Algunas reflexiones sobre los retos de un futuro inmediato. Rosario, Homo Sapiens Ediciones.

Appel, K.-O. (1991). Teoría de la verdad y ética del discurso. Barcelona, Paidós.

Arzubialde, S. (2009). Ejercicios espirituales de S. Ignacio. Historia y análisis (Vol. 1). Sal Terrae.

Bauman, Z. (2005). Los retos de la educación en la modernidad líquida. Barcelona, Editorial Gedisa. (2013). Sobre la educación en un mundo líquido: conversaciones con Riccardo Mazzeo. Buenos Aires, Argentina: Paidós.

Beytía, J. C. (2008). La cultura juvenil y sus desafíos. Revista de Espiritualidad Ignaciana, (117), 11-25.

Buroz Echenagucia, O., S.J. Identidadignaciana: un modo de ser, recuperado de: http://www.cerpe.org.ve/ tl_files/Cerpe/contenido/documentos/Pastoral\%20-\%20Gene$\mathrm{ral} /$ San\%20lgnacio\%20y\%20Espiritualidad\%20lgnaciana/2\%20 IDENTIDAD\%20IGNACIANA\%20 UN\%20MODO\%20DE\%20SER.pdf

Castells, M., Flecha, R., Freire, P. et alt. (2001). Nuevas perspectivas críti- cas en educación, Barcelona, Ed. Paidós.

Colomer, T. (1997). La enseñanza y el aprendizaje de la comprensión lectora. Revista Signos, (20), 6-15.

Conferencia de Provinciales de América Latina -CPAL. (2016). La importancia de la Narrativa: La comunicación en la CG36, recuperado de: http://www.cpalsj.org/la-importancia-de-la-narrativa-la-comunicacion-en-la-cg36/

Congregación General XXXIV de la Compañía de Jesús. (2013), recuperado de: http://www.cpalsj.org/ wp-content/uploads/2013/03/ CG34-Decretos.pdf

Congregación General XXXV de la Compañía de Jesús, recuperado de: http://www.sjweb.info/35/documents/Decretos.pdf

Congregaciones Generales, Post Vaticano II. (2016), recuperado de: http://www.cpalsj.org/wp-content/uploads/2016/04/Doc-01Jose-Maria-Arena-SJ.-Chile.pdf

Cortina, A. (sf). "Ética discursiva y educación en valores", recuperado de: http://www.insumisos. com/lecturasinsumisas/Etica\%20 discursiva\%20y\%20valores.pdf 
Grammatico. G. (2008). Experiencia de la palabra y del silencio. España: Fundación Dialnet.

Heidegger, M. (2004). Lógica. La pregunta por la verdad. Madrid, Alianza Editorial.

Herrán, M. T. y Restrepo, J.D. (2005). Ética para periodistas. Bogotá, Grupo Editorial Norma.

Lellouche, R. (2001). El fundamento de la moral y la "ética del discurso" de Karl-Otto Apel. En Teoría política y comunicación (pp. 200-232). Barcelona, Gedisa.

Liévanos-Álvarez, M. C. (2016). La gestión del conocimiento en la construcción colectiva del proyecto curricular de centro, elemento para la innovación educativa (Master's thesis, ITESO).

Lúquez, P., Fernández, O., \& Bustos, C. (2015). Formación ética del estudiante universitario socialmente responsable. Omnia, 20(2).

Man-Ging, C., Merchán, M., Racines, F. (2010). Hacia una integración de la pastoral juvenil, Centro de Publicaciones de la PUCE, Ideaz.

Mercer, N. (2001). Palabras y mentes. Cómo usamos el lenguaje para pensar juntos. Barcelona, Paidós.

Martínez de Toda, J., S.J. (2011). Contextualización histórica del Sector Comunicación en la Compañía de Jesús de América Latina y el
Caribe, recuperado de: http:// jesuitascam.org/wp-content/ uploads/2012/02/Contextualizaci\%C3\%B3n.pdf

Merchán, M. y Zary, P. (2016). “Estrategias didácticas en el aprendizaje de la lengua y el fortalecimiento de competencias lingüísticas y de comunicación interpersonal", en Pucará Revista de Humanidades. Cuenca, Facultad de Filosofía, Letras y Ciencias de la Educación.

Navarro, R. F. (2015). La investigación de la comunicación en América Latina: condiciones y perspectivas para el siglo XXI. Comunicación y sociedad, (36).

O' Malley, J. W., S.J. (1993). Los primeros jesuitas. Bilbao, Ediciones Mensajero.

Poblete, M. Bezanilla, M.J., Fernández, D., Campo, L. (2016). Formación del docente en competencias genéricas: un instrumento para su planificación y desarrollo, en Educar Departament de Pedagogia Aplicada de la UAB, vol 52/1 pp. 7191, recuperado de: http://educar. uab.cat/article/view/v52-n1-poblete-bezanilla-fern\%C3\%A1ndez-campo/713-pdf-es

Racines, F. (2004). Apuntes para la incorporación de la Espiritualidad Ignaciana en la P.U.C.E.-Manabí, Portoviejo, mimeo. 
(2016). "El Pensamiento Ignaciano y la Universidad Contemporánea" en Pensamiento Ciencia Sociedad. Quito, Pontificia Universidad Católica del Ecuador.

Reig, D. (2012), Estudiantes, autonomía y aprendizaje aumentado: ¿escuelas y docentes como actores clave para otorgar(les) sentido?, recuperado de http://encuentro. educared.org/group/hacia-lasescuelas-3-0-y-los-estudiantes-3-0/page/dolors-reig (2012). Sociedad aumentada y aprendizaje, videoconferencia, recuperado de: https://youtu. be/6-F9L9avcwo

(2013). Los jóvenes en la era de la hiperconectividad, recuperado de: http://www.dreig.eu/caparazon/2013/05/17/jovenes-en-la-era-de-la-hiperconectividad/

Reker, G. T., \& Woo, L. C. (2011). Personal Meaning Orientations and Psychosocial Adaptation in Older Adults. SAGE Open, 1(1). doi: 10.1177/2158244011405217

Rui-Wamba, M.A. (s.f.) Espiritualidad como componente de la identidad ignaciana, recuperado de: http://www.ausjal.org/tl_files/ ausjal/images/contenido/Documentos/Publicaciones/Identidad\%20y\%20Mision/Espirituali-
dad,\%20Componente\%20de\%20 la\%20ldentidad\%20Ignaciana\%20-\%20Miguel\%20Angel\%20 Rui-Wamba\%20S.J\%20_1_.pdf

Ruiz, V. M. (2014). Habilidades para la vida: una propuesta de formación humana. Itinerario Educativo, 28(63), 61-89.

Velasco, L., S. J. (2014). El rol actual del profesor en la universidad jesuita (Vol. 1). ITESO.

Villa, A., Poblete, M. (directores), (2005). Aprendizaje basado en competencias. Una propuesta para la evaluación de las competencias genéricas. Bilbao, Universidad de Deusto.

Villa, A. (2011) Modelo de innovación en la educación superior: un enfoque para el desarrollo y la evaluación en la Innovación, recuperado de: http://www.foroinnovacionuniversitaria.net/archives/2011/10/ Ponencia-Aurelio-Villa.pdf

Villa, A., Campo, L., Villa, O., Arranz, S. y García, A. (2014). Valoración del profesorado de magisterio sobre el aprendizaje basado en competencias implantado, Profesorado, Revista de currículum y formación del profesorado, vol 17, recuperado de: http://www.ugr. es/ recfpro/rev173ART2.pdf

Villa, A. \& García, A. (2014). Un sistema de garantía de calidad de la do- 
cencia: un estudio de caso. Revis- Von Foerster, H. (2010). Construyendo ta Electrónica Interuniversitaria una realidad en Watzlawick y de Formación del Profesorado, vol 17 (3), pp. 65-78, recuperaotros. La realidad inventada. Bardo de: http://www.aufop.com/ celona, Gedisa.

aufop/uploaded_files/articulos/1415234669.pdf 